\title{
In Vitro Mitigation of Pathogenic Bacteria and Virulence Factors Using a Hydroconductive Dressing
}

\author{
Lauren T. Moffatt ${ }^{1}$, Rachel T. Ortiz ${ }^{1}$, Bonnie C. Carney ${ }^{1}$, Rachael M. Bullock ${ }^{1}$, Martin C. Robson ${ }^{2}$, \\ Marion H. Jordan ${ }^{1,3}$, Jeffrey W. Shupp ${ }^{1,3}$ \\ ${ }^{1}$ Firefighters' Burn and Surgical Research Laboratory, MedStar Health Research Institute, Washington DC, USA \\ ${ }^{2}$ Department of Surgery, University of South Florida, Tampa, USA \\ ${ }^{3}$ The Burn Center, Department of Surgery, MedStar Washington Hospital Center, Washington DC, USA \\ Email: jeffrey.w.shupp@medstar.net
}

Received October 7, 2013; revised October 31, 2013; accepted November 8, 2013

Copyright (C) 2013 Lauren T. Moffatt et al. This is an open access article distributed under the Creative Commons Attribution License, which permits unrestricted use, distribution, and reproduction in any medium, provided the original work is properly cited.

\begin{abstract}
Wound infections can have devastating effects on healing as well as the health of the patient. Complications increase when the pathogens are capable of producing virulence factors and/or are drug resistant. Novel methods are needed to take on the challenges of treating such wounds. Drawtex ${ }^{\circledR}$ dressing is purported to have hydroconductive properties that allow it to draw away debris and exudate from the wound into the dressing. The goal of this work is to better define these interactions of this experimental dressing with bacteria and virulence factors. Two series of in vitro experiments were performed. First, pieces of experimental dressing were submerged in a series of cultures in flasks and samples of the dressing and cultures were taken over 90 minutes and assayed for bacteria and virulence factor levels. Second, experimental or standard care (control) dressings were placed on selective agar plated with pathogens of interest. Dressings and the agar covered by them were used to quantify bacteria and virulence factors over time. The experimental dressing took up both bacteria and virulence factors to a larger extent than the control dressing. Experimental dressing significantly reduced the load of bacteria and virulence factors in cultures compared to control culture without dressing. Based on the ability of the dressing to take up bacteria and virulence factors in this study, the data point to the potential for this dressing to be similarly effective in reducing or eliminating pathogen from wounds, potentially increasing the chances of successful wound healing.
\end{abstract}

Keywords: Infection; MRSA; Virulence Factors; Dressing

\section{Introduction}

Wound infections significantly increase the risk of patient morbidity and mortality [1,2], especially in instances where the pathogens in question are able to produce exoproteins in the wound environment that increase their virulence [3]. Many strains of pathogenic bacteria commonly associated with wounds are capable of producing an array of these virulence factors [4-8] (Table 1). These factors such as hemolysins, collagenases, leukocidins, and exotoxins can make the infection more invasive, derail the local healing process, exacerbate the inflammatory response, and cause bacteremia and systemic immune disruption [9-11]. Because of the incredible influence that virulence factors have on pathogenicity, recent drug developments have included the oxazoladinones and glycylcyclines, aimed via various mechanisms at inhibiting protein synthesis and therefore abrogating ability to produce virulence factors $[12,13]$. While this has led to potential improvements in treating infections, increasingly, strains of pathogenic bacteria observed in

Table 1. Wound-relevant bacteria species and associated virulence factors.

\begin{tabular}{cc}
\hline $\begin{array}{c}\text { Bacteria } \\
\text { Species }\end{array}$ & Virulence Factors \\
\hline MRSA & $\begin{array}{r}\text { Toxic shock syndrome toxin 1 (TSST-1), } \\
\text { Panton-Valentine leukocidin (PVL), Staphylococcal } \\
\text { enterotoxins (SEB, SEA) }\end{array}$ \\
$\begin{array}{c}\text { Pseudomonas } \\
\text { aeruginosa } \\
\text { Klebsiella } \\
\text { pneumoniae } \\
\text { Acinetobacter } \\
\text { baumannii }\end{array}$ & Exotoxin A, Phospholipase C \\
\hline
\end{tabular}


hospitalized patients as well as in outpatients, are multidrug resistant, presenting a new set of challenges in pharmacotherapy.

Many topical agents and dressing products are aimed at preventing infection by creating a barrier against contamination, or have been designed to be bactericidal in nature, often impregnated with antimicrobials such as silver $[14,15]$. Drawtex ${ }^{\circledR}$ is a novel hydroconductive dressing product that has been recently developed and is reported to remove large quantities of exudate, bacteria, and debris from wounds. In multiple case reports and small clinical studies, it has been shown to decrease slough, granulation tissue, and eschar in wound beds [16].

While multiple observations of the positive impact of this dressing on healing potential have been documented, little work has been done in a controlled model system to systematically test its capabilities in mitigating bacteria and virulence factor presence. Preliminary experiments in controlled model systems that were aimed at determining the capabilities of Drawtex ${ }^{\circledR}$ in taking up bacteria and protein from wounds and media were performed and demonstrated that the dressing is in fact capable of moving significant amounts of both protein (albumin) and bacteria (Methicillin-resistant Staphylococcus aureus, MRSA) from media and wounds [16]. The reduction of pathogen and virulence factor load in wounds will impact the local healing dynamic processes and immune response, potentially removing the infectious complication and allowing the progression of healing. If the dressing can achieve this efficiently, it may succeed in advancing wound healing and reducing pathogenicity of infection, where antibiotics and topical bactericidal agents may fall short.

In order to further characterize the ability of Drawtex ${ }^{\circledR}$ to interact with wound-relevant pathogens and their associated virulence factors, a series of in vitro experiments were designed. Based on preliminary data, it was hypothesized that the dressing will be able to effectively decrease levels of bacteria and virulence factors in media. Experiments were designed using liquid media in flasks to demonstrate the absorption capabilities of the dressing when saturated. A second set of experiments were designed on solid agar media to more closely mimic a "wound" surface and determine the ability of the dressing to inhibit bacterial proliferation. The results described here point to the potential for the experimental dressing to serve a critical role in the management of wounds as it may help mitigate invasive infections and perhaps more importantly, reduce the pathogenicity of the infection by reducing the presence of virulence factors.

\section{Methods}

\subsection{Bacterial and Virulence Factor Absorption}

To determine how effectively the experimental dressing can take up wound-relevant bacteria species and their associated virulence factors from media, three sterile flasks containing $50 \mathrm{ml}$ of Todd Hewitt (TH) Broth (BD Biosciences, Franklin Lakes, NJ) were prepared. Two of these flasks were then inoculated with methicillin-resistant Staphylococcus aureus (MRSA) and incubated at $37^{\circ} \mathrm{C}$ in a shaker overnight until the cultures contained approximately $1 \times 10^{8} \mathrm{CFU} / \mathrm{ml}$ of MRSA. The strain of MRSA used is a previously characterized clinical isolate, known to produce multiple virulence factors. The third flask served as a control, was not inoculated, and contained $50 \mathrm{ml}$ of $\mathrm{TH}$ broth only. Two pieces of the experimental dressing $\left(\right.$ Drawtex $^{\circledR}$ ) were cut to $3 \mathrm{~cm} \times 3 \mathrm{~cm}$, with one piece submerged into one of the two MRSA culture flasks and the other submerged in the uninoculated TH broth (Figure 1). The second inoculated flask was left without dressing.

The flasks were subsequently incubated at $37^{\circ} \mathrm{C}$ with gentle rocking for a total of 90 minutes, with samples of submerged dressing and media from each flask collected at $1,10,30,45,60$, and 90 minutes post dressing submergence. Culture samples consisted of $0.5 \mathrm{ml}$ aliquots drawn from the flasks and then frozen at $-80^{\circ} \mathrm{C}$, or used immediately in quantitative cultures, while dressing samples were obtained using a $2 \mathrm{~mm}$ punch biopsy and were weighed, flash frozen and stored at $-80^{\circ} \mathrm{C}$. MRSA and associated virulence factors of interest, specifically toxic shock syndrome toxin 1 (TSST), Panton-Valentine leukocidin (PVL), and alpha hemolysin (AH), were quantified in these samples using quantitative culture or ELISA methods described below.

This experiment was performed in triplicate $(n=3)$ and was then repeated using similarly prepared flasks containing tryptic soy broth (Sigma-Aldrich, St. Louis, MO) with approximately $1 \times 10^{8} \mathrm{CFU} / \mathrm{ml}$ of Pseudomonas aeruginosa, Klebsiella pneumoniae, or Acinetobacter baumannii. For each species, associated virulence factors were quantified (Table 2). Statistically significant differences between virulence factor and bacteria levels in cultures absent of submerged dressing (controls) versus levels in cultures containing dressing, were assessed using unpaired t-tests with a significance level of $\mathrm{p}<0.05$. The third flasks containing uninoculated media and dressing were assayed as described and no bacteria or toxin were detected for any experiment at any time point (data not presented). Data were plotted using GraphPad Prism (GraphPad, La Jolla, CA, Version 6.02).

\subsection{Bacterial and Virulence Factor Inhibition}

To examine the impacts of the experimental dressing on both bacterial growth and virulence factor production in a controlled in vitro system, Staphylococcus aureus-selective Mannitol salt agar (MSA) plates (BD Biosciences, Franklin Lakes, NJ) were seeded with $100 \mu \mathrm{l}$ of $\mathrm{TH}$ 


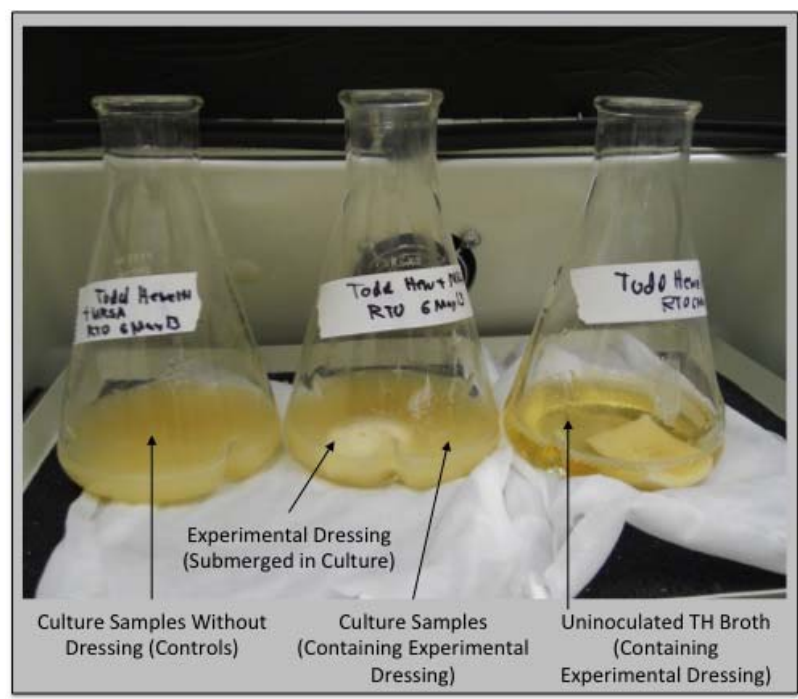

Figure 1. Absorption experimental design. Flasks prepared with media, with or without bacterial inoculation and with or without submerged experimental dressing, used in the bacteria and virulence factor absorption experiments. Media and dressing samples from each flask were taken over a time course with levels of bacteria and virulence factors quantified.

Table 2. Species and virulence factors studied in the present work.

\begin{tabular}{cc}
\hline Bacteria Species Studied & Virulence Factors Examined \\
\hline MRSA & TSST-1, PVL, AH \\
Pseudomonas aeruginosa & Exotoxin A \\
Klebsiella pneumoniae & Endotoxin \\
Acinetobacter baumannii & Endotoxin \\
\hline
\end{tabular}

Broth containing $1 \times 10^{6} \mathrm{CFU} / \mathrm{ml}$ of MRSA. Experimental dressing $\left(\right.$ Drawtex $\left.^{\circledR}\right)$ or Mepilex ${ }^{\circledR}$ (Molnlycke, Norcross, GA), used as a standard of care (SOC) control dressing, were cut to $3 \mathrm{~cm} \times 3 \mathrm{~cm}$ and placed in the center of the inoculated MSA plates. Once daily from day 0 (placement of the dressing) to day 4, $2 \mathrm{~mm}$ punch biopsies of the dressings and the agar fields covered by them (Figure 2) were collected, weighed, flash frozen, stored at $-80^{\circ} \mathrm{C}$ and subsequently used to quantify bacterial levels and virulence factors produced by the pathogen.

Digital photos of the plates were taken each day. The experiment was performed in triplicate $(n=3)$. This experiment was repeated using Leeds Acinetobacter (LA) plates (Hardy Diagnostics, Santa Maria, CA) seeded with Acinetobacter baumannii, MacConkey agar plates (BD Biosciences, Franklin Lakes, NJ) seeded with Klebsiella pneumoniae, and Pseuduomonas-selective agar plates (Thermo Fisher Scientific, Waltham, MA) seeded with Pseudomonas aeruginosa. Statistical analysis was performed using unpaired t-tests $(\mathrm{p}<0.05)$ to determine differences in bacteria and toxin levels contained in the

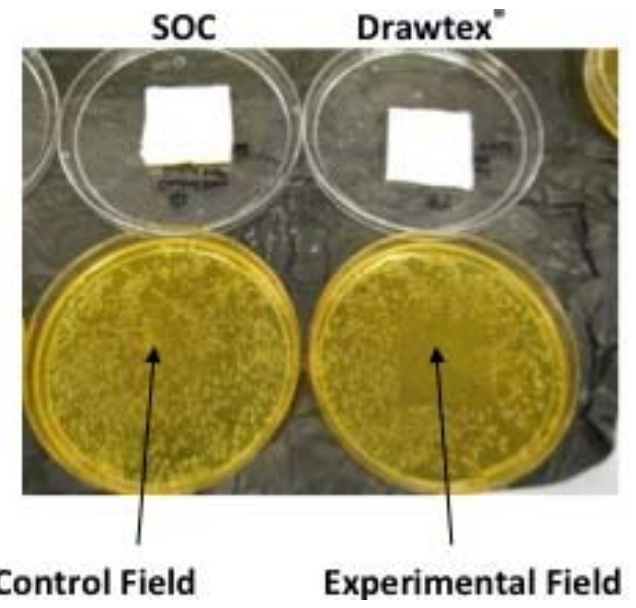

Figure 2. Inhibition experimental design. Media plates used for assessing bacteria and virulence factor inhibition of experimental dressing, as compared to a standard of care (SOC). Bacteria were plated and experimental or control dressings were subsequently applied. Samples of the dressings and the agar fields covered by them were sampled over a time course and bacteria and virulence factors quantified.

experimental versus control dressings (and corresponding agar fields covered by them) and data were plotted using GraphPad Prism.

\subsection{Quantitative Cultures}

Dressing and agar samples were weighed, flash frozen in liquid nitrogen and then stored at $-80^{\circ} \mathrm{C}$. The flash frozen samples were homogenized using a TissueLyser (Qiagen, Germantown, MD), reconstituted with sterile phosphate buffered saline (PBS), and vortexed. Culture samples were assayed immediately after withdraw from flasks. The homogenates or freshly obtained culture samples were serially diluted with $100 \mu 1$ of each dilution plated on selective media plates. Plate selection depended upon experimental bacteria; MSA plates selective for S. aureus for MRSA quantification (yellow colonies indicating coagulase positivity and presumptive $S$. aureus), LA plates selective for Acinetobacter species (light pink colonies, indicating alkaline products and presumptive Acinetobacter), Pseudomonas-selective plates for Pseudomonas species (inhibition of growth of other bacterial species), or MacConkey agar plates to indicate Klebsiella species (pink colonies indicating lactose fermentation and presumptive Klebsiella). Plates were incubated at $37^{\circ} \mathrm{C}$ for 24 hours, colonies of the appropriate color were counted, and colony-forming units (CFUs) per gram of agar or dressing, or per milliliter of media were calculated.

In the absorption experiment (in flasks), fold changes in $\mathrm{CFU} / \mathrm{g}$ in dressing and $\mathrm{CFU} / \mathrm{ml}$ in media over time were calculated compared to data at $\mathrm{t}=1$ minute postsubmergence. 


\subsection{Virulence Factor Quantification}

Enzyme-linked immunosorbent assays (ELISAs) were used to quantify TSST-1, PVL, AH, and Pseudomonas Exotoxin A (PEA). Dressing and agar samples were collected, preserved and processed as described above with homogenates reconstituted in PBS with $0.5 \%$ Tween 20 (PBST). Aliquots of $100 \mu \mathrm{l}$ were added to wells of 96 well immunoassay plates (Nalge Nunc International, Rochester, NY) coated with $100 \mu \mathrm{l}$ of a $1 \mathrm{mg} / \mathrm{ml}$ primary antibody raised to TSST-1, AH (ToxinTechnology, Inc., Sarasota, FL), PVL (Integrated Biotherapeutics, Gaithersburg, MD), or PEA (Sigma-Aldrich, St. Louis, MO). Culture samples and a serial dilution of standard purified TSST-1, PVL, AH, or PEA were treated in the same way. The plates containing samples and standard curve were then incubated at $37^{\circ} \mathrm{C}$ for 2 hours, washed with PBST, and then $100 \mu \mathrm{l}$ of secondary antibody diluted 1:300 in PBST was added to each well. The plates were placed on a shaker and incubated at $37^{\circ} \mathrm{C}$ for 1 hour and then washed with PBST. Each well then received $100 \mu \mathrm{l}$ of 2,2'-Azino-bis(3-ethylbenzothiazoline-6-sulfonate) (ABTS) with $0.05 \mathrm{M}$ phosphate citrate buffer (Sigma-Aldrich) and hydrogen peroxide. The plates were sealed and incubated
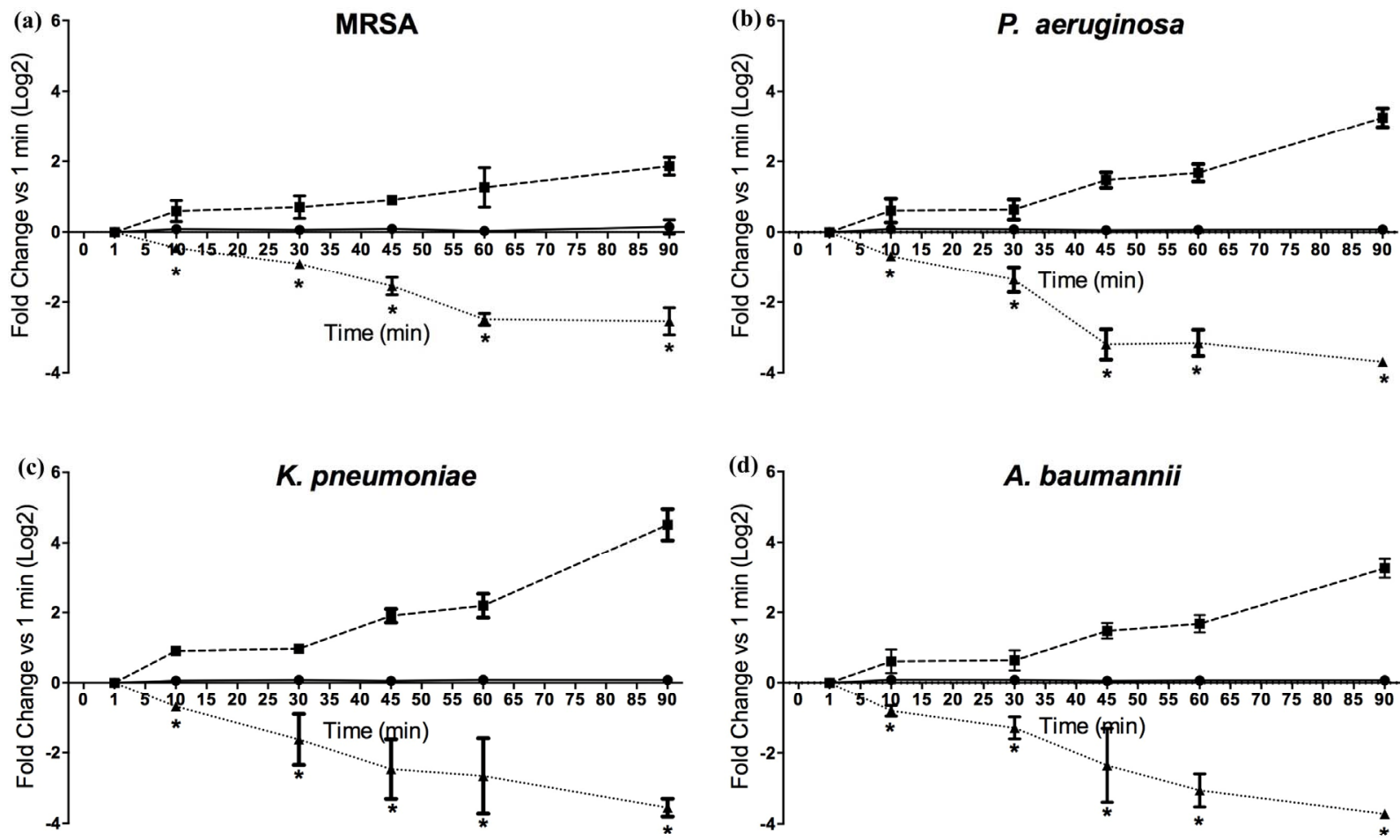

$\rightarrow$ Culture Samples Without Dressing (Control)
... Culture Samples (Containing Experimental Dressing)

Figure 3. Fold change from bacteria levels quantified at 1 minute post-dressing submergence in cultures or dressing samples over time. Comparisons in bacteria levels between cultures containing experimental dressing versus cultures without dressing were made at each time point using an unpaired t-test $(p<0.05)$ with statistically significant difference indicated $\left({ }^{*}\right)$. at room temperature in the dark, and then $100 \mu \mathrm{l}$ of $0.5 \%$ of Sodium dodecyl sulphate (SDS) in distilled water stopped the reaction. The plates were read at $405 \mathrm{~nm}$ in a VICTOR Multilabel Counter (PerkinElmer, Waltham, MA) and Workout 2.0 (PerkinElmer) was used to process the results.

A. baumannii endotoxin (AE) and K. pneumonia endotoxin (KE) were both quantified in culture samples and dressing and agar samples (processed as above) using a Pierce LAL Chromogenic Endotoxin Quantitation Kit (Thermo Fisher Scientific, Rockford, IL).

\section{Results}

\subsection{Bacterial and Virulence Factor Absorption}

Bacteria were not detected in any of the baseline dressing samples (pre-submergence), or in the uninoculated $\mathrm{TH}$ broth throughout the time course. Starting at 10 minutes post-dressing submergence, all cultures that contained submerged experimental dressing had a significantly lower bacterial count compared to the control culture without dressing (Figure 3). A decreasing trend in bacteria levels in the cultures containing dressing continued over the

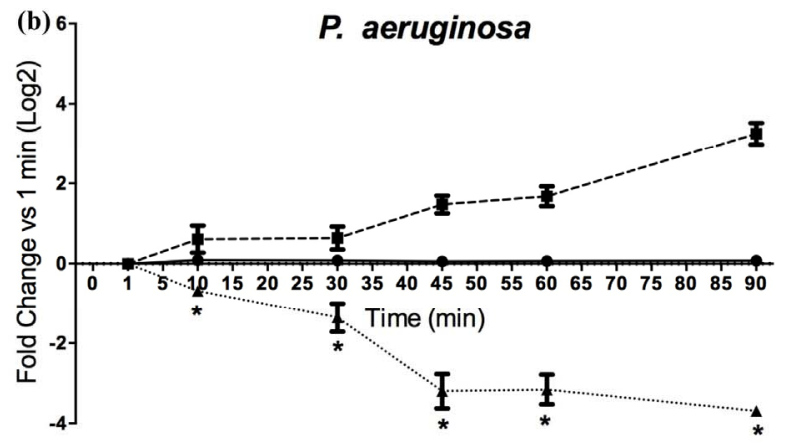

- Experimental Dressing Samples (in Culture) 
90 minute time course, while at the same time, bacteria quantified in the dressing samples increased correspondingly (Figure 3).

TSST1 levels in the MRSA culture containing experimental dressing were significantly lower compared to the control culture (without dressing) by 30 minutes postdressing submergence (Figure 4(a)). At 45 minutes into the time course, $\mathrm{AH}, \mathrm{PVL}, \mathrm{KE}$, and AE levels quantified in cultures of associated bacteria containing the dressing were significantly lower compared to the control cultures (Figures 4(b)-(f) respectively). By 60 minutes, PEA was also significantly lower in dressing-containing Pseudomonas cultures compared to the control. Similar to the trends in bacterial levels in the dressing submerged in cultures, virulence factor levels also showed an increase over time, as levels in the media decreased (Figure 4).
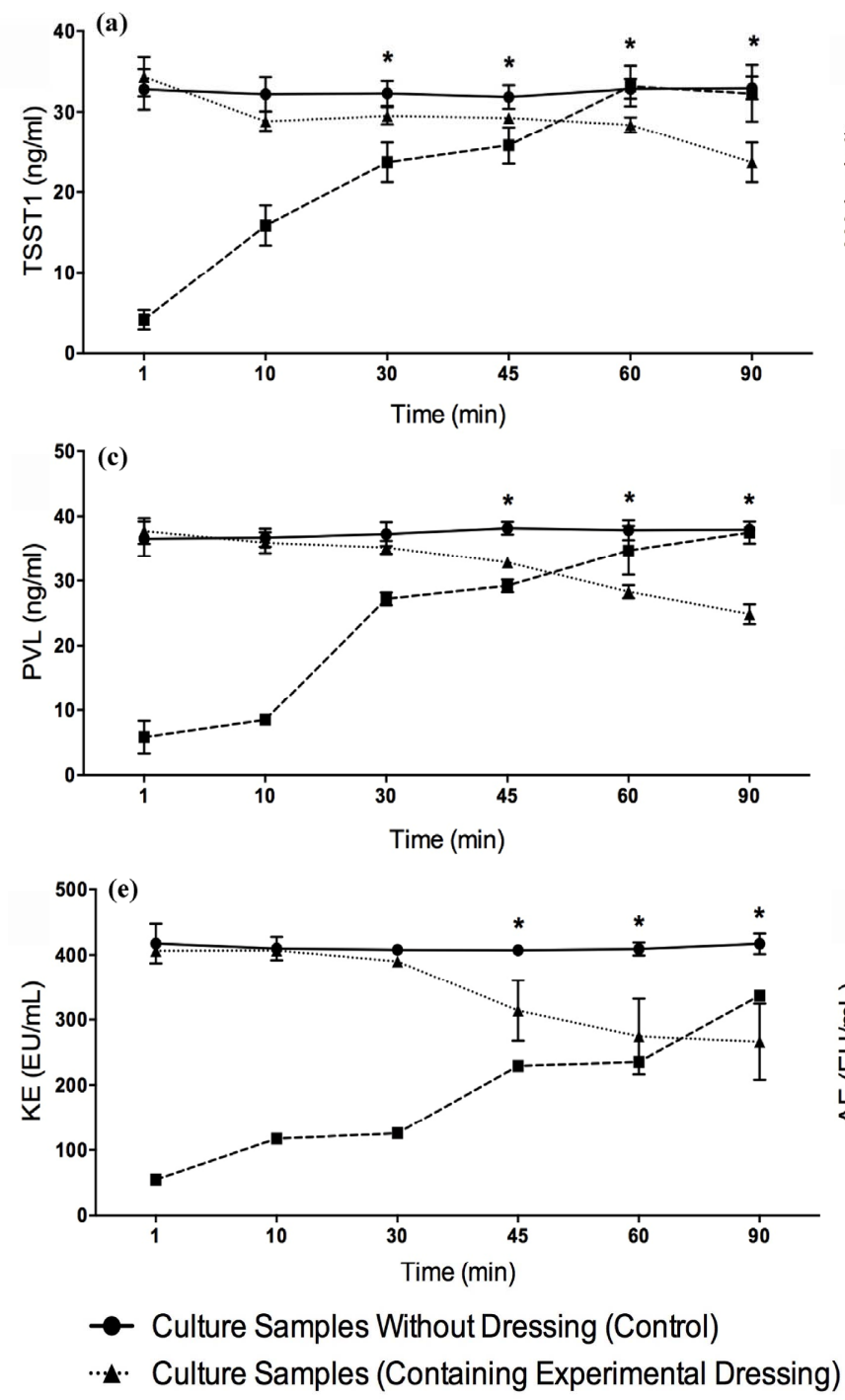

\subsection{Bacterial and Virulence Factor Inhibition}

Upon examination of the inoculated plates for all species of pathogen studied, noticeable differences existed beginning 24 hours after placement of the dressings on the inoculated plates (study day 1) in the number of colonies in agar fields covered with SOC control dressing compared to experimental dressing-covered fields (digital images not shown). Beginning on day 1 of the experiment, this difference was statistically significant in plates seeded with MRSA and $P$. aeruginosa (Figures 5(a) and (b)), while the difference in bacteria quantified in experimental fields was significantly lower than that in control fields by day 2 for $K$. pneumoniae and $A$. baumannii (Figures 5(c) and (d)). Decreasing trends in bacteria continued through day 4 and was similar for all
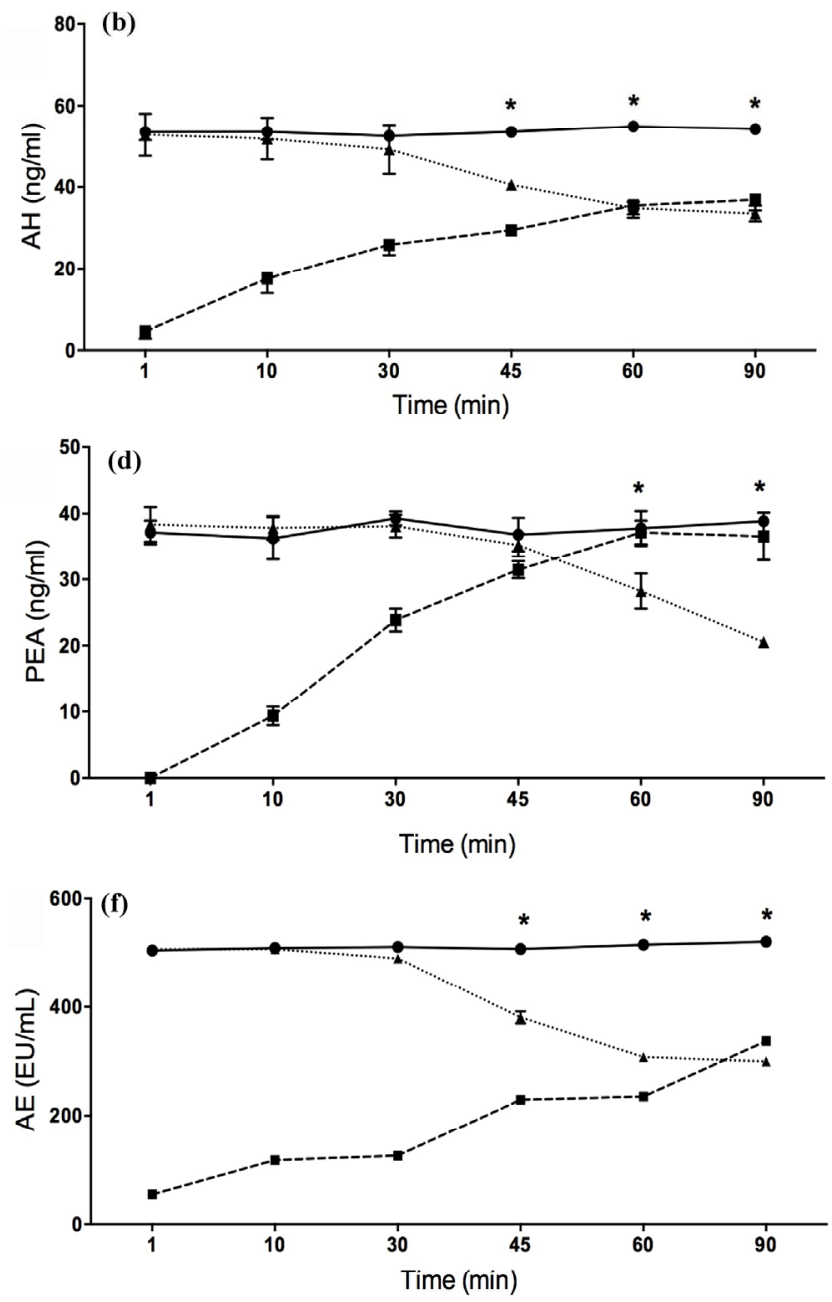

Experimental Dressing Samples (in Culture)

Figure 4. Levels of TSST1 (a), AH (b), PVL (c), PEA (d), KE (e), or AE (f) quantified in culture or dressing samples over a 90 minute time course in the absorption experiments. Statistically significant $\left(^{*}\right)$ differences in levels in culture samples containing dressing as compared to cultures without dressing were determined at each time point using an unpaired $t$-test $(p<0.05)$. 

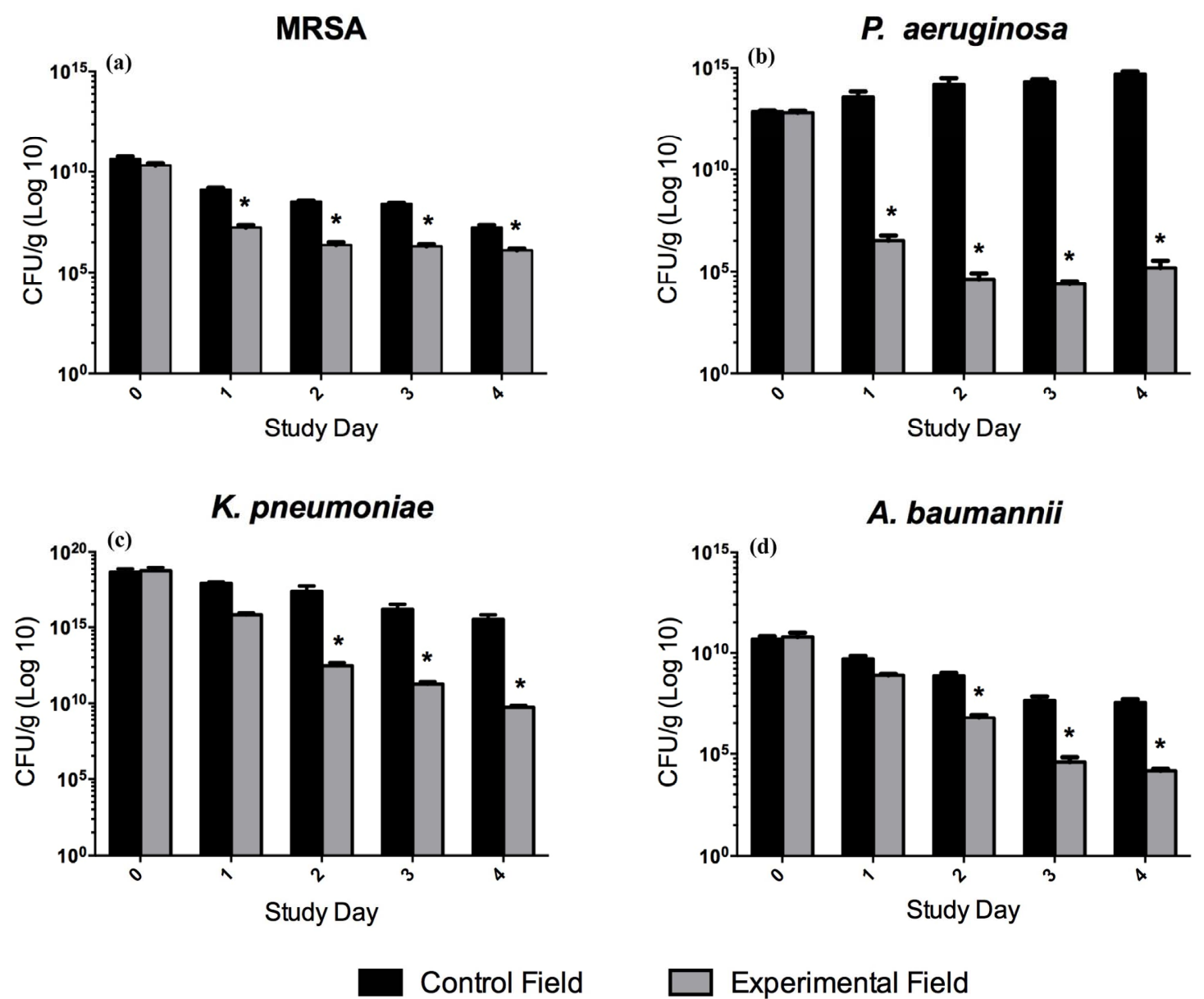

Figure 5. Colony forming units of bacteria per gram (CFU/g) of inoculated agar samples covered with either experimental or control dressings, quantified over 4 days. Statistically significant differences $\left(^{*}\right)$ in bacteria levels between experimental dressing-covered agar and control dressing (SOC)-covered agar samples at each time point were assessed using an unpaired t-test $(\mathbf{p}<0.05)$.

species of pathogen examined (Figure 5). While the agar fields covered with experimental dressing had lower levels of bacteria than those covered with control dressing, at the same time there was a significantly higher level of bacteria quantified in the experimental dressing compared to the control dressing at all time points tested and for all species (Figure 6).

Virulence factor levels were also reduced in the agar covered by experimental dressing compared to the control dressing-covered agar. Some virulence factors (TSST1, PVL, and PEA) became non-detectable in experimental agar fields early in the time course (Figures 7(a), (c) and (d)) while control dressings were not capable of reducing any of the factors below detection limits within the four day experiment (Figure 7). For those virulence factors not already below detection limits in experimental agar fields, levels were significantly lower as compared to controls by day 2 (AE) or 3 (AH and KE) (Figures 7(b), (e) and (f) respectively). As patterned in the bacteria data, virulence factor levels showed a trend of increase in the experimental dressing over the time course, as levels in the agar fields decreased (Figure 8). Experimental dressing had significantly higher levels of TSST1 and PVL than the control dressing by study day 1 (Figures 8(a) and (c)) and this was also true for levels of PEA (Figure 8(d)). Levels of AE quantified in experimental dressing were significantly greater than those in control dressings by day 2 (Figure 8(f)), while AH and KE levels were significantly higher by day 3 (Figures 8(b) and (e)).

\section{Discussion}

The first set of experiments described here was designed to examine the ability of the experimental dressing to take up both bacteria and virulence factors from bacterial culture. A consistently lower level of both bacteria and virulence factors was quantified in the cultures that contained submerged experimental dressing when com- 
MRSA

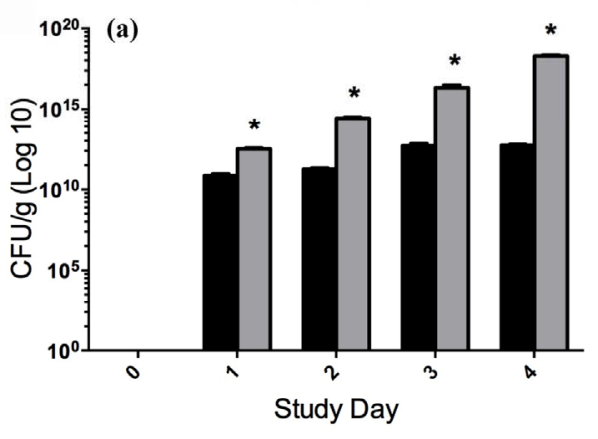

K. pneumoniae

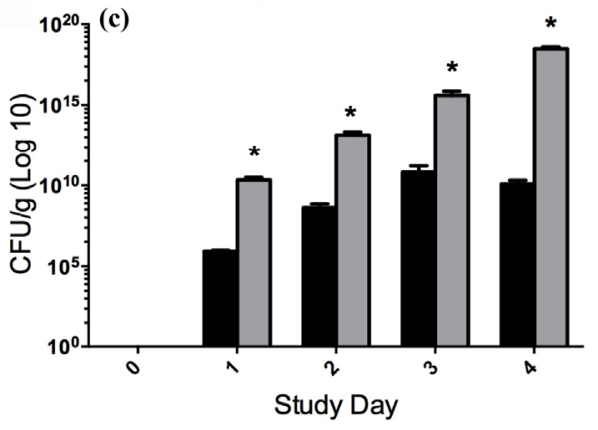

Control Dressing
P. aeruginosa

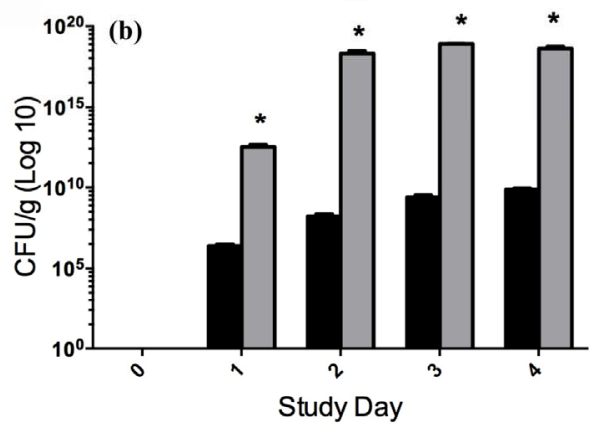

A. baumannii

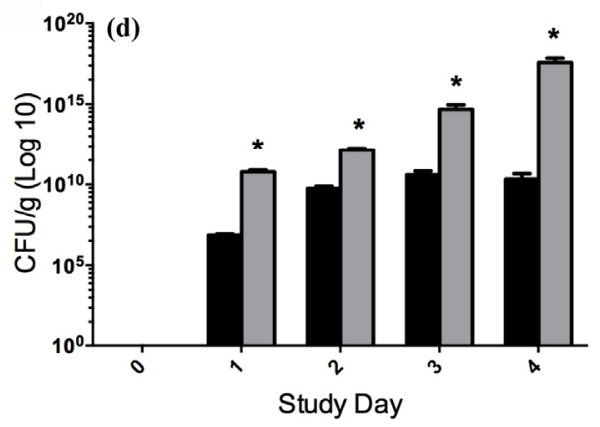

Experimental Dressing

Figure 6. Colony forming units of bacteria per gram (CFU/g) of either experimental or SOC control dressing after 1, 2, 3, or 4 days of covering an agar field inoculated with bacteria. Statistically significant differences $\left({ }^{*}\right)$ in bacteria levels between experimental dressing and control dressing samples at each time point were assessed using an unpaired t-test $(p<0.05)$.
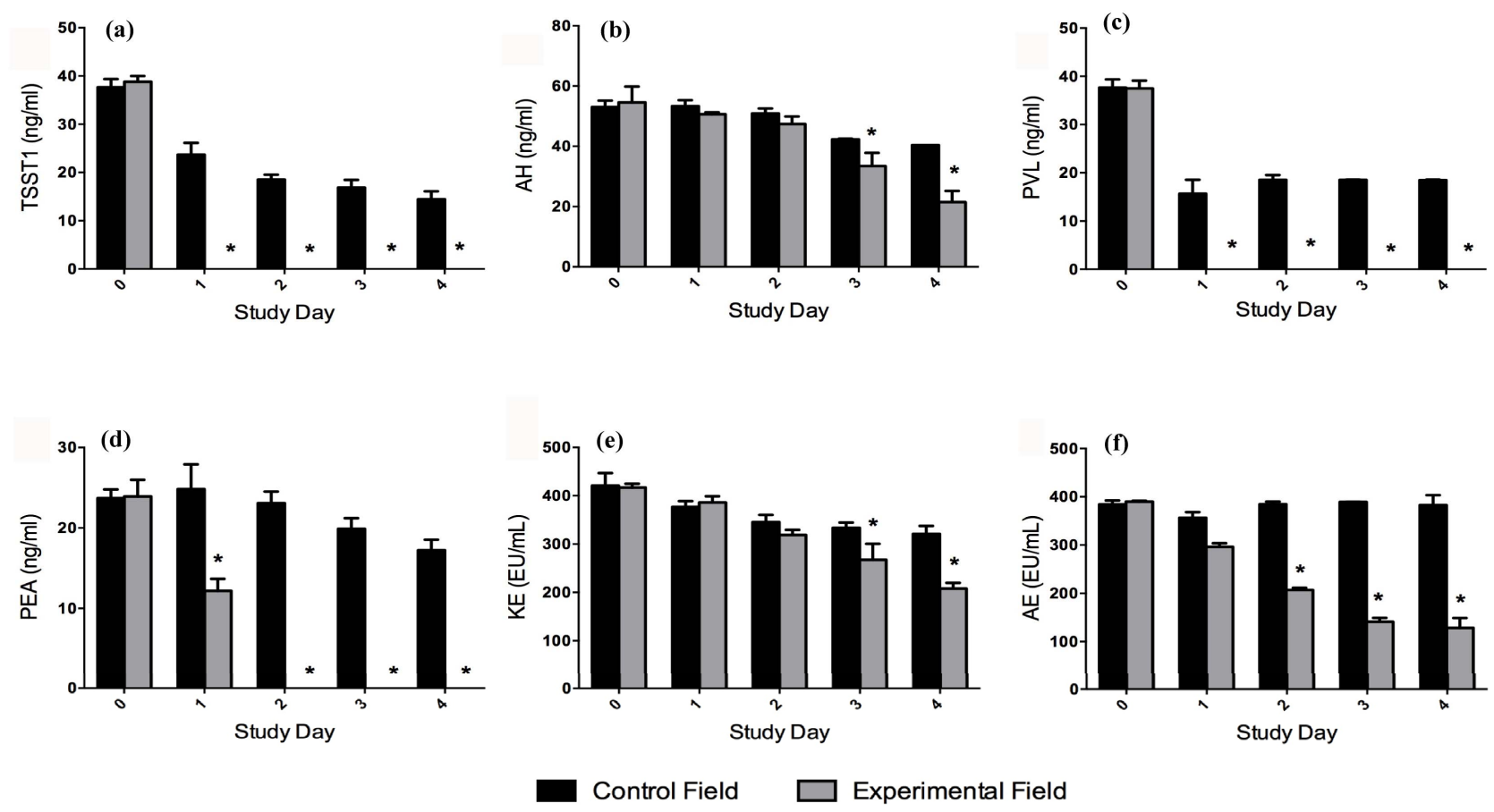

Figure 7. Levels of TSST1 (a), AH (b), PVL (c), PEA (d), KE (e), or AE (f) quantified in inoculated agar samples covered with either experimental or control dressings, quantified over 4 days. Statistically significant $\left(^{*}\right)$ differences in levels in control samples as compared to experimental samples were determined at each time point using an unpaired t-test $(p<0.05)$. 

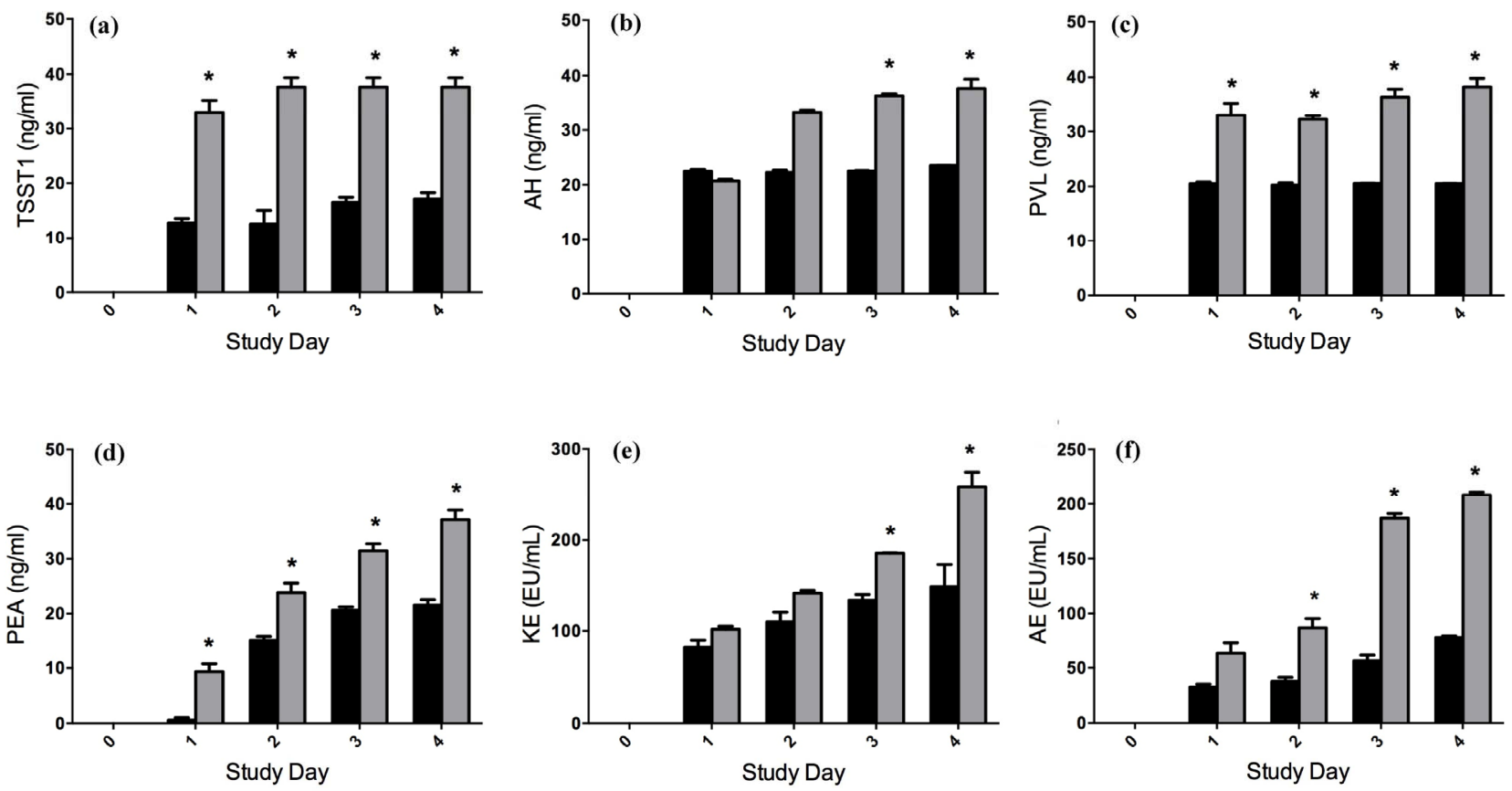

Control Dressing

Experimental Dressing

Figure 8. Levels of TSST1 (a), AH (b), PVL (c), PEA (d), KE (e), or AE (f) quantified in experimental or SOC control dressing after 1, 2, 3, or 4 days of covering an agar field inoculated with bacteria. Statistically significant $\left(^{*}\right)$ differences in levels in control dressing as compared to experimental dressing were determined at each time point using an unpaired $t$-test $(p<0.05)$.

pared to the control cultures that did not contain any dressing (Figure 3). At the same time, there was a consistent increase over the time course in bacteria and virulence factors quantified in the dressing itself throughout the study (Figures 3 and 4). This demonstrates a clear capability to absorb both gram positive and gram negative pathogens and a variety of proteinaceous virulence factors, indicating a potential role in removing the same contaminants from wound exudate and fluids. Moreover, this hints at interesting mechanisms of action that allow the dressing material to accumulate materials with diverse structural and biochemical properties over time.

The second set of experiments described demonstrated a significant mitigation of gram positive and gram negative bacterial presence, as well as reduced virulence factor levels, in plates seeded with pathogens of interest (Figures 5 and 7). Compared to an SOC control dressing, the experimental dressing appeared able to accumulate pathogen and protein of interest to a larger extent (Figures 6 and 8), and in turn, eliminate both to a higher degree from the agar. As these data show, over a period of days the dressing is capable of steadily accumulating additional pathogen and virulence factors. If the experiment had been taken out for a longer time period, it would be hypothesized that the dressing may reach a "saturation" point, perhaps dependent on the bacterial load present on the media. Whether this would occur before all detectable bacteria or toxin was eliminated from the media, it is a reasonable question, and is likely dependent on the amount of pathogen and virulence factor present when the dressing is applied. If in fact a maximum capacity exists for the dressing, further questions would include whether the contaminants taken up by the dressing would eventually then be released back into the agar; or rather, how long they can be retained. Further experiments should be done to evaluate this, as clinical implications may exist related to frequency of dressing changes and whether the presence of contaminants in the dressing would, over some amount of time, impact the wound being treated.

It should also be noted that while Drawtex ${ }^{\circledR}$ showed much more significant impacts on pathogen and virulence factor mitigation in the plated agar models, the SOC control did demonstrate similar trends of decrease in bacteria and pathogen in the fields covered, though to a much more minimal extent and not for all of the bacteria and virulence factors examined.

The goal of the work described here was to characterize the efficacy of this experimental dressing in abrogating multiple types of bacteria and the associated virulence factors in vitro. To date, no research has been done to characterize the interactions of this new dressing with common wound pathogens, and the protein products that result in increased pathogenicity. This work should serve 
as a foundation for further controlled studies, both in vitro and in vivo, to fully understand the capabilities of this promising dressing in treating wounds.

\section{Acknowledgements}

This work was funded in part by SteadMed Medical and by the DC Firefighters' Burn Foundation.

\section{REFERENCES}

[1] J. R. Ebright, "Microbiology of Chronic Leg and Pressure Ulcers: Clinical Significance and Implications for Treatment," The Nursing Clinics of North America, Vol. 40, No. 2, 2005, pp. 207-216. http://dx.doi.org/10.1016/j.cnur.2004.09.003

[2] C. E. Black and J. W. Costerton, "Current Concepts Regarding the Effect of Wound Microbial Ecology and Biofilms on Wound Healing," The Surgical Clinics of North America, Vol. 90, No. 6, 2010, pp. 1147-1160. http://dx.doi.org/10.1016/j.suc.2010.08.009

[3] G. Schiavo and F. G. van der Goot, "The Bacterial Toxin Toolkit," Nature Reviews Molecular Cell Biology, Vol. 2, No. 7, 2001, pp. 530-537.

http://dx.doi.org/10.1038/35080089

[4] M. Otto, "Basis of Virulence in Community-Associated Methicillin-Resistant Staphylococcus aureus," Annual Review of Microbiology, Vol. 64, 2010, pp. 143-162. http://dx.doi.org/10.1146/annurev.micro.112408.134309

[5] M. Li, et al., "Evolution of Virulence in Epidemic Community-Associated Methicillin-Resistant Staphylococcus aureus," Proceedings of the National Academy of Sciences of the United States of America, Vol. 106, No. 14, 2009, pp. 5883-5888. http://dx.doi.org/10.1073/pnas.0900743106

[6] I. Pastar, et al., "Interactions of Methicillin Resistant Staphylococcus Aureus USA300 and Pseudomonas Aeruginosa in Polymicrobial Wound Infection," PloS One, Vol. 8, No. 2, 2013, p. e56846. http://dx.doi.org/10.1371/journal.pone.0056846

[7] V. S. Nikbin, et al., "Molecular Identification and Detection of Virulence Genes among Pseudomonas aeruginosa Isolated from Different Infectious Origins," Iranian Journal of Microbiology, Vol. 4, No. 3, 2012, pp. 118-123.
[8] R. El Fertas-Aissani, et al., "Virulence Profiles and Antibiotic Susceptibility Patterns of Klebsiella pneumoniae Strains Isolated from Different Clinical Specimens," $\mathrm{Pa}$ thologie-Biologie, 2012.

http://dx.doi.org/10.1016/j.patbio.2012.10.004

[9] P. M. Schlievert, "Cytolysins, Superantigens, and Pneumonia Due to Community-Associated Methicillin-Resistant Staphylococcus aureus," Journal of Infectious Diseases, Vol. 200, No. 5, 2009, pp. 676-678. http://dx.doi.org/10.1086/605333

[10] J. M. Yarwood, D. Y. Leung and P. M. Schlievert, "Evidence for the Involvement of Bacterial Superantigens in Psoriasis, Atopic Dermatitis, and Kawasaki Syndrome," FEMS Microbiology Letters, Vol. 192, No. 1, 2000, pp. 1-7. http://dx.doi.org/10.1111/j.1574-6968.2000.tb09350.x

[11] S. Bi, et al., "The Cellular and Molecular Immune Response of the Weanling Piglet to Staphylococcal Enterotoxin B," Experimental Biology and Medicine (Maywood), Vol. 234, No. 11, 2009, pp. 1305-1315. http://dx.doi.org/10.3181/0901-RM-2

[12] P. M. Schlievert and J. A. Kelly, "Clindamycin-Induced Suppression of Toxic-Shock Syndrome-Associated Exotoxin Production," Journal of Infectious Diseases, Vol. 149, No. 3, 1984, p. 471. http://dx.doi.org/10.1093/infdis/149.3.471

[13] J. W. Shupp, et al., "Treatment with an Oxazolidinone Antibiotic Inhibits Toxic Shock Syndrome Toxin-1 Production in MRSA-Infected Burn Wounds," Journal of burn Care \& Research: Official Publication of the American Burn Association, Vol. 34, No. 2, 2013, pp. 267-273. http://dx.doi.org/10.1097/BCR.0b013e318280e35a

[14] A. Lansdown, et al., "Silver Dressings: Absorption and Antibacterial Efficacy," Nursing Times, Vol. 101, No. 46, 2005, pp. 45-46.

[15] P. Aramwit, et al., "In Vitro Evaluation of the Antimicrobial Effectiveness and Moisture Binding Properties of Wound Dressings," International Journal of Molecular Sciences, Vol. 11, No. 8, 2010, pp. 2864-2874. http://dx.doi.org/10.3390/ijms11082864

[16] M. Robson, "Innovations for Wound Bed Preparation: The Role of Drawtex Hydroconductive Dressings," Proceedings of a Symposium of Investigators, Tampa, 4 May 2012. 